\title{
DISEÑO Y CONSTRUCCIÓN DE ACUMULADOR SOLAR TÉRMICO POR CAMBIO DE FASE PARA UN SECADOR SOLAR FAMILIAR
}

\author{
DESIGN AND CONSTRUCTION OF PHASE CHANGE SOLAR \\ THERMAL ACCUMULATOR FOR A FAMILY SOLAR DRYER
}

\author{
Kléber Janampa-Quispe ${ }^{i^{*}([)}$, Octavio Cerón-Balboa' ${ }^{1}$ \\ Oswaldo Morales-Morales ${ }^{1}\left[\right.$, Julio Oré-García ${ }^{1}$ (])
}

${ }^{1}$ Facultad de Ingeniería de Minas, Geología y Civil, Universidad Nacional de San Cristóbal de Huamanga, Ayacucho, Perú

Recibido (Recieved): 17/02/2020 Aceptado (Accepted): 05/03/2020

\begin{abstract}
RESUMEN
Se diseña y construye un acumulador solar térmico mediante el principio de cambio de fase, para optimizar el proceso de secado en un secador solar familiar, en un periodo adicional al cese de la incidencia de la radiación solar; el material de cambio de fase seleccionado es la parafina comercial, cuyas propiedades térmicas han sido caracterizados mediante el medidor KD2 Pro. La temperatura de fusión de la parafina $\left(53^{\circ} \mathrm{C}\right)$ se encuentra en torno a la temperatura de operación del secador solar de mediana temperatura. El acumulador solar térmico se caracteriza por ser modular, formado por doce unidades cilíndricas de aluminio reciclado, donde cada unidad contiene $200 \mathrm{~g}$ de parafina e incorpora mallas con aletas de aluminio para mejorar el flujo de calor al exterior y optimizar su transferencia al aire. Como resultado se ha logrado aumentar el tiempo adicional de secado en un orden de $2 \mathrm{~h}$ sin intervención de la radiación solar, manteniendo una temperatura del aire mayor a $30{ }^{\circ} \mathrm{C}$ previamente sometido a una radiación solar media de $533 \mathrm{Wm}^{-2}$.
\end{abstract}

Palabras Clave: acumulador térmico, material de cambio de fase.

\section{ABSTRACT}

A solar thermal accumulator is designed and built using the phase change principle, to optimize the drying process in a familiar solar dryer, in an additional period to the cessation of the incidence of solar radiation; the phase change material selected is the commercial paraffin, whose thermal properties have been characterized by the KD2 Pro meter. The melting temperature of the paraffin $\left(53^{\circ} \mathrm{C}\right)$ is around the operating temperature of the medium temperature solar dryer. The solar thermal accumulator is characterized by being modular, consisting of twelve cylindrical units of recycled aluminum, where each unit contains $200 \mathrm{~g}$ of paraffin and incorporates meshes with aluminum fins to improve the flow of heat to the outside and optimize its transfer to the air. As a result, the additional drying time has been increased by an order of 2 hours without intervention of solar radiation, maintaining an air temperature greater than $30^{\circ} \mathrm{C}$ previously subjected to an average solar radiation of $533 \mathrm{Wm}^{-2}$.

Keywords: thermal accumulator, phase change material.

\footnotetext{
${ }^{*}$ Corresponding author.:

E-mail: kleber.janampa@unsch.edu.pe 


\section{INTRODUCCIÓN}

La tecnología solar, tiene entre sus mayores limitaciones la disponibilidad intermitente de la energía, lo que genera un rendimiento inestable y diseños poco confiables [1]. Esta inestabilidad, hace que durante el proceso de secado en los secadores solares, se generen problemas como el secado incompleto del producto, la condensación nocturna de la humedad dentro del secador, haciendo perder parte del secado logrado en el día [2]. Por lo que es necesario desarrollar alternativas que permitan alargar el proceso de secado durante horas que no haya radiación solar, acumulando la energía solar en horas de sol. Los acumuladores solares térmicos con materiales de cambio de fase son una alternativa para contrarrestar la inestabilidad temporal de la energía solar. En el presente trabajo de investigación se ha diseñado y construido un sistema de almacenamiento de energía térmica solar para optimizar el proceso de secado en un secador solar de uso familiar en horas donde ya no hay la intervención solar; el sistema dispone de unidades modulares cilíndricos de aluminio obtenidos de materiales reciclados. Cada unidad se llena de la parafina como material de cambio de fase. El sistema acumula en total $2.4 \mathrm{~kg}$ de parafina, con el que se ha logrado aumentar el tiempo de secado en un orden de $2 \mathrm{~h}$ adicionales, a la interrupción de la radiación solar, manteniendo una temperatura del aire sobre los $30^{\circ} \mathrm{C}$.

\section{ANTECEDENTES}

Actualmente, los acumuladores solares térmicos que generan interés, son los acumuladores de cambio de fase (PCM), dado que presenta una mayor densidad energética acumulada en comparación a los acumuladores de calor sensible y presenta mayor estabilidad en la temperatura. Un material PCM que abunda en nuestra localidad, utilizado tradicionalmente en la cerería artesanal, es la parafina, cuyas propiedades térmicas son adecuadas para aplicaciones térmicas solares de mediana temperatura, entre $53{ }^{\circ} \mathrm{C}$ a $61{ }^{\circ} \mathrm{C}$ [3] que corresponde a la temperatura de fusión de la parafina.

En Colombia, se ha implementado un sistema de acumulación térmica con parafina como material de cambio de fase, encapsulados en tubos de cobre, para un secador de túnel tipo Hohenheim [1]. De igual manera Glogower [2], desarrolla un sistema de almacenamiento de energía térmica con aceite de coco como material PCM, encapsulado en material de aluminio, para un secador tipo Hohenheim, logrando un secado más rápido. En Chile, el Dr. Alejandro Reyes [4] publicó parte de sus resultados sobre el diseño y evaluación de un intercambiador de calor que utiliza parafina como PCM y utiliza latas de bebida reciclados para acumular la energía solar. Reyes, destaca la baja conductividad térmica de la cera de parafina, que dificulta la remoción de calor desde las latas.

\section{METODOLOGÍA}

El acumulador térmico se basa en el principio de acumulación por calor latente de la parafina, que durante el periodo de incidencia de radiación solar acumula energía térmica. El intercambio de calor acumulado con el aire como fluido caloportador, se optimiza sobre la base del principio de los intercambiadores de calor.

Para ello, se ha caracterizado las propiedades térmicas de la parafina comercial local. Luego, se dimensiona la estructura geométrica del acumulador que se caracteriza por ser modular y hecha en base a materiales reciclados.

La disponibilidad energética del acumulador, está orientado a atender la demanda energética de un secador solar familiar de mediana temperatura durante horas que se interrumpe la incidencia de la radiación solar. Se evalúa comparativamente el comportamiento térmico del secador solar cuando incorpora el acumulador y cuando no lo incorpora.

\subsection{PARÁMETROS TÉRMICOS DE LA PARAFINA}

La tabla I muestra la densidad en fase sólida y líquida de la parafina utilizada como material PCM.

TABLA I

Densidad de la parafina comercial

\begin{tabular}{lcc}
\hline & Fase sólida & Fase líquida $\left(70^{\circ} \mathrm{C}\right)$ \\
\hline $\mathrm{m}(\mathrm{g})$ & 220.92 & 220.92 \\
$\mathrm{~h}(\mathrm{~cm})$ & 9.8 & 11.2 \\
$\mathrm{r}(\mathrm{cm})$ & 2.86 & 2.86 \\
$\mathrm{~V}\left(\mathrm{~cm}^{3}\right)$ & 251.82 & 287.80 \\
$\delta\left(\mathrm{g} / \mathrm{cm}^{3}\right)$ & 0.877 & 0.768 \\
\hline \multicolumn{2}{l}{ Nota: Se puede observar que la densidad de la } \\
parafina en fase líquida es $12 \%$ menor que en \\
fase sólida.
\end{tabular}

Se utiliza el medidor térmico KD2 Pro, que se basa en el método de fuente de calor de la línea transitoria, para medir la conductividad térmica (k), el calor específico volumétrico $(C)$, resistencia térmica $(\rho)$ y difusividad (D) de la parafina comercial utilizado como PCM del acumulador.

TABLA II

Conductividad térmica de la parafina. Sensor KS1

\begin{tabular}{ccc}
\hline $\mathrm{k}(\mathrm{W} / \mathrm{m} \mathrm{K})$ & $\rho\left({ }^{\circ} \mathrm{C} \mathrm{cm} / \mathrm{W}\right)$ & $\mathrm{T}\left({ }^{\circ} \mathrm{C}\right)$ \\
\hline 0.192 & 506.4 & 23.08 \\
0.194 & 516.7 & 21.11 \\
0.193 & 518.2 & 20.24 \\
0.193 & 519.2 & 20.04 \\
0.193 & 519.1 & 20.050 \\
\hline
\end{tabular}

TECNIA Vol.30 ํํ1 Enero-Junio 2020 


\begin{tabular}{ccc}
\hline 0.192 & 521.3 & 20.12 \\
0.192 & 521.8 & 20.22 \\
0.192 & 520.5 & 20.29 \\
\hline 0.193 & 517.9 & 20.64 \\
\hline Nota: & La temperatura de & medición de la
\end{tabular}

conductividad térmica es de $20.64^{\circ} \mathrm{C}$.

De la tabla II, la conductividad térmica de la parafina comercial resulta:

$$
k=0.193 \pm 0.01 \frac{W}{m K} \quad,(1)
$$

TABLA III

Calor específico volumétrico y difusividad de la parafina. Sensor $\mathrm{SH} 1$

\begin{tabular}{lcc}
\hline $\mathrm{D}\left(\mathrm{mm}^{2} / \mathrm{s}\right)$ & $\mathrm{C}\left(\mathrm{MJ} / \mathrm{m}^{3} \mathrm{~K}\right)$ & $\mathrm{T}\left({ }^{\circ} \mathrm{C}\right)$ \\
\hline 0.157 & 1.852 & 25.74 \\
0.159 & 1.823 & 25.05 \\
0.162 & 1.783 & 24.06 \\
0.164 & 1.748 & 23.28 \\
0.159 & 1.717 & 24.15 \\
0.164 & 1.738 & 23.21 \\
0.165 & 1.723 & 22.71 \\
0.167 & 1.704 & 22.17 \\
\hline 0.162 & 1.761 & 23.80 \\
\hline
\end{tabular}

Nota: A partir del calor específico volumétrico se puede obtener el calor específico de la muestra.

De la tabla III, la difusividad térmica y el calor específico de la parafina comercial es:

$$
\begin{aligned}
& D=0.162 \pm 0.016 \frac{\mathrm{mm}^{2}}{\mathrm{~s}}, \\
& C=1.761 \pm 0.176 \frac{\mathrm{MJ}}{\mathrm{m}^{3} \mathrm{~K}},
\end{aligned}
$$

Para determinar la temperatura de fusión y solidificación de la parafina, se toma una muestra de $11.18 \mathrm{~g}$ que se coloca en una probeta al interior del agua contenido en un vaso precipitado, que va calentándose lentamente. La curva de calentamiento (Fig. 1), muestra que la temperatura de fusión de la parafina comercial está en el orden de $53^{\circ} \mathrm{C}$.

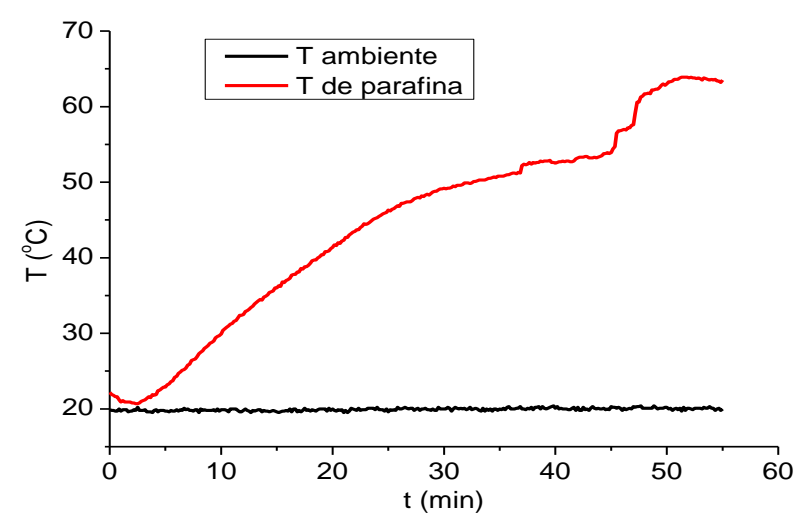

Fig. 1. Variación de la temperatura durante el proceso de calentamiento de la parafina (11.18 g), para determinar su temperatura de fusión.
La Fig. 2, ilustra la variación de la temperatura en el interior de una unidad de acumulación, la que muestra la temperatura de fusión y solidificación de la parafina en torno a $53^{\circ} \mathrm{C}$.

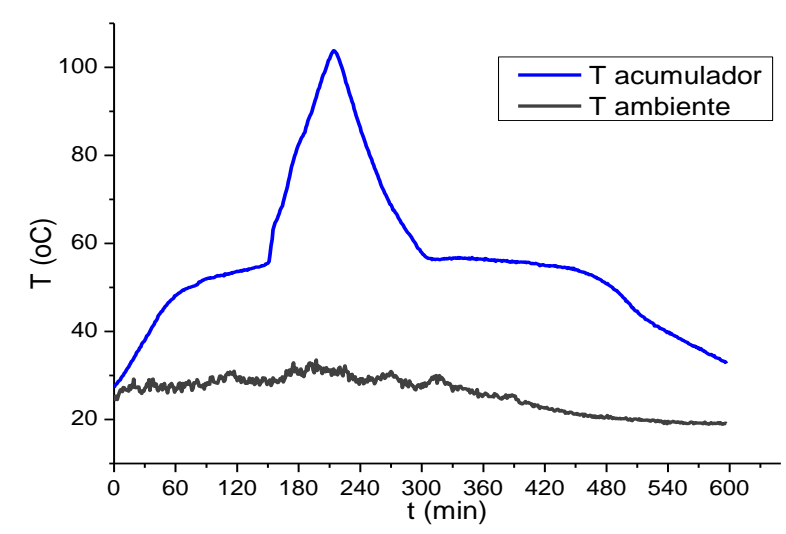

Fig. 2. Curva de calentamiento y enfriamiento de la parafina en el interior de la unidad de acumulación con malla metálica.

\subsection{DISEÑO DEL ACUMULADOR DE CAMBIO DE FASE}

El acumulador térmico se compone de unidades básicas que se comportan como intercambiadores independientes de calor entre el material PCM y el aire. El carácter modular del acumulador permite disponer del número de unidades necesarias según el requerimiento energético de la aplicación específica. Las unidades del acumulador son de forma cilíndrica cuyas dimensiones son $2.7 \mathrm{~cm}$ de radio y $12 \mathrm{~cm}$ de altura (Fig. 3), que contienen $200 \mathrm{~g}$ de parafina como material de cambio de fase. Dado que la conductividad térmica de la parafina es de $0.2 \mathrm{Wm}^{-1}{ }^{\circ} \mathrm{C}^{-1}$ [5] que no facilita la conducción térmica a través de la cera, se construye una malla metálica de aluminio de manera que une el interior de la cera con la superficie del cilindro, para mejorar el proceso de conducción térmica.

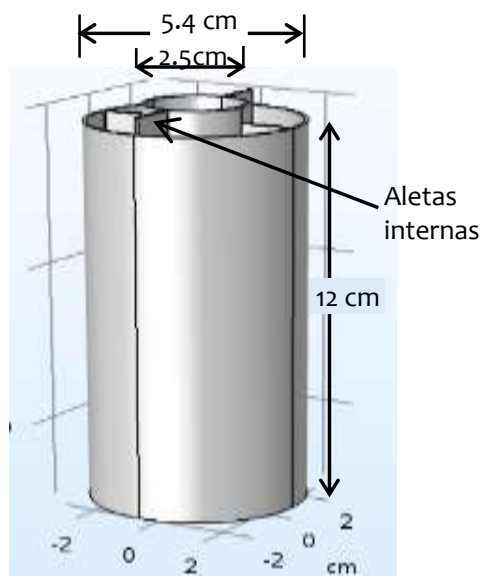

Fig. 3. Dimensión del prototipo de la unidad del acumulador de cambio de fase de forma cilíndrica y de estructura en aluminio. 
El sistema de acumulación térmica con PCM de parafina, consta de 12 unidades, distribuidas simétricamente como muestra la Fig. 4.

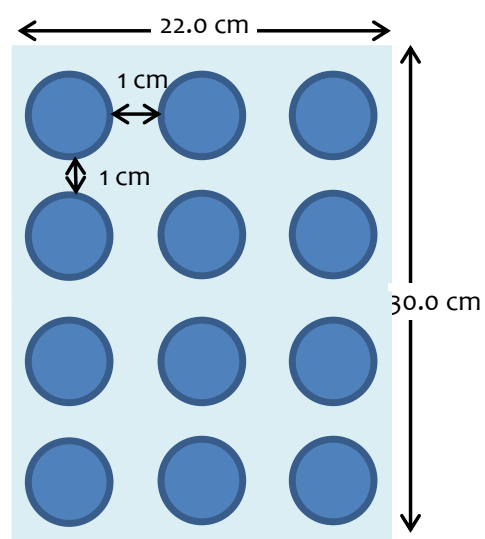

Fig. 4. Distribución de las unidades en un sistema de acumulación térmica: vista superior.

Tomando de base el objetivo de lograr diseños sencillos, materiales de menor costo y ambientalmente viables, se utilizan latas de aluminio reciclados para la construcción de las unidades del acumulador, las cuales incorporan una malla metálica ubicada concéntricamente en el interior del cilindro y unida mediante aletas con la superficie interior de la lata.

Las unidades se llenan de parafina líquida y una vez secas se cierran lo más herméticamente posibles y se pinta la superficie lateral externa con negro mate, como ilustra la Fig. 5.

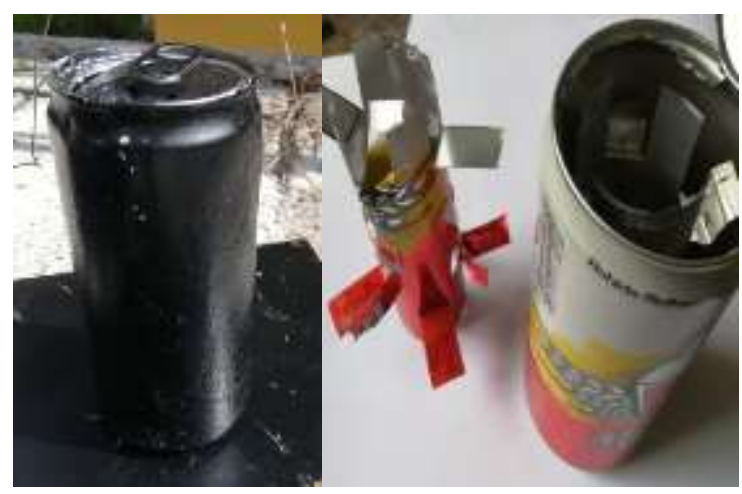

Fig. 5. Unidades del acumulador de parafina y malla metálica de aluminio; unidad pintada de negro mate.

El sistema o módulo de acumulación térmico tiene 12 unidades, que se ubican sobre una placa metálica, distribuyéndolas simétricamente a $1 \mathrm{~cm}$ de cada una en 3 columnas por 4 filas (Fig. 6).

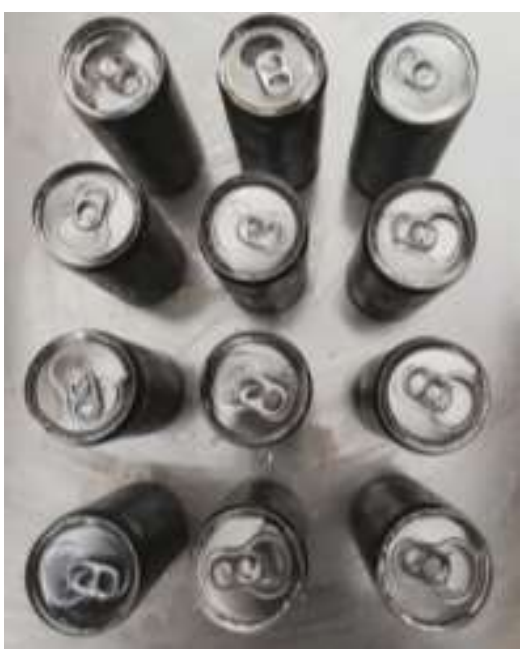

Fig. 6. Acumulador solar térmico con PCM (Parafina), doce unidades instaladas en el colector del secador.

Para determinar el espaciamiento óptimo entre las latas del acumulador, se simula el comportamiento térmico de dos unidades cilíndricas mediante el software COMSOL MULTIPHYSICS 5.3; estableciendo condiciones estacionarias para el intercambio térmico por convección, conducción y radiación entre la superficie de la lata y el aire como fluido caloportador.

La Fig. 7. muestra la variación de la temperatura del aire entre las unidades cilíndricas de acumulación térmica en función de la distancia entre las superficies lateral de los cilindros. Se encuentra que cuanto menor es el espaciamiento mayor es la temperatura del aire entre ellas, no obstante aumentaría la resistencia al flujo del aire. Considerando criterios prácticos de construcción se elige $1 \mathrm{~cm}$ como la distancia más adecuada.

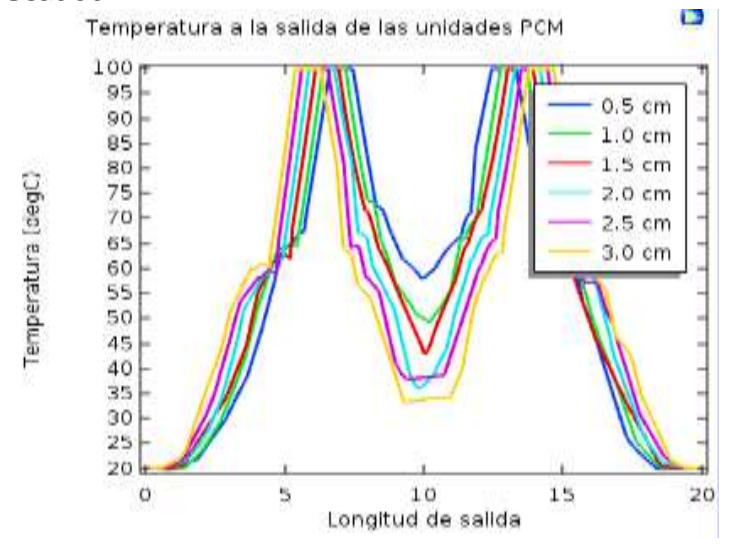

Fig. 7. Variación de la temperatura del aire entre $0.5 \mathrm{~cm}$ a $3 \mathrm{~cm}$ de distancia entre las latas. Simulación COMSOL MULTIPHYSICS 5.3.

\subsection{SECADOR SOLAR}

El secador solar, en la que se implementa el módulo del acumulador térmico, es de tipo cabina (Fig. 8) que dispone de un colector solar plano de $0.72 \mathrm{~m}^{2}$. La cabina, para el secado indirecto de productos dispone las bandejas en niveles para optimizar el secado; el flujo de aire caliente ingresa por la base de la cabina y 
el aire húmedo sale a través de un tubo en la parte superior que genera el efecto tiro.

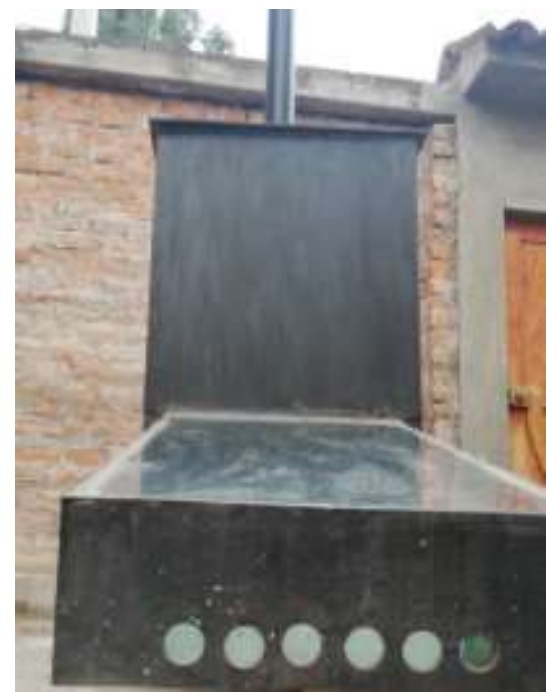

Fig. 8. Secador solar Indirecto-Pasivo.

Para dimensionar el requerimiento de energía necesario para secar un determinado producto, se asume la temperatura ambiente media de $18{ }^{\circ} \mathrm{C}$ y humedad relativa media de $64 \%$ correspondiente al mes de marzo (Centro Meteorológico de la UNSCH). EI proceso de secado indirecto, considera el calentamiento del aire hasta $40{ }^{\circ} \mathrm{C}$, para luego intercambiar energía con el producto haciendo que el aire se enfría adiabáticamente hasta la saturación, correspondiendo la temperatura final del aire $19.3{ }^{\circ} \mathrm{C}$, obtenido de las cartas psicrométricas para la zona, con la que se tiene una capacidad de remoción de agua de hasta $8.4 \mathrm{~g}$ de agua/kg de aire. La tabla IV, muestra la cantidad de energía necesaria para secar lúcuma y papa cocida a escala familiar. La humedad en base húmeda inicial y final de la lúcuma se obtuvo experimentalmente; en tanto que para la papa se tomó la referencia de la guía para el desarrollo de proyectos de secado solar en comunidades rurales [6].

TABLA IV

Dimensionamiento de la energía necesaria para el secado de Lúcuma у рара

\begin{tabular}{lccc}
\hline Característica & & Lúcuma & Papa \\
\hline Peso del material sin secar $(\mathrm{kg})$ & $\mathrm{W}_{\mathrm{o}}$ & 1.000 & 1.000 \\
$\begin{array}{l}\text { Humedad en base húmeda inicial } \\
\left.\text { (kgagua/ } / \mathrm{kg}_{\text {prod hum }}\right)\end{array}$ & $\mathrm{M}_{\mathrm{wb} 1}$ & 0.667 & 0.75 \\
$\begin{array}{l}\text { Humedad en base húmeda final } \\
\text { (kgagua/kg prod hum) }\end{array}$ & $\mathrm{M}_{\mathrm{wb} 2}$ & 0.093 & 0.130 \\
Tiempo & $\mathrm{t}$ & 5 & 5 \\
Cantidad de agua retirada $(\mathrm{kg}$ agua/ $/ \mathrm{h})$ & $\mathrm{W}_{\mathrm{a}}$ & 0.127 & 0.143 \\
Humedad absoluta del aire: entrada & $\mathrm{w}_{1}$ & 0.0117 & 0.0117 \\
Humedad absoluta del aire: salida & $\mathrm{w}_{2}$ & 0.0201 & 0.0201 \\
Consumo de aire seco $(\mathrm{kg} / \mathrm{h})$ & $\mathrm{m}_{\mathrm{a}}$ & 15.072 & 16.968 \\
Entalpía del aire: entrada & $\mathrm{h}_{1}$ & 47.7 & 47.7 \\
Entalpía del aire: salida & $\mathrm{h}_{2}$ & 70.4 & 70.4 \\
Energía consumida $(\mathrm{Kj} / \mathrm{h})$ & $\mathrm{Q}_{\mathrm{a}}$ & 342.1 & 385.2 \\
\hline
\end{tabular}

Nota: La humedad en base húmeda inicial y final de la lúcuma, se obtuvo con un analizador de humedad Ohaus MB35.

Elaboración propia.

\section{ANÁLISIS DE RESULTADOS}

\subsection{ENERGÍA ACUMULADA Y SECADO}

El comportamiento térmico de una unidad del acumulador que contiene parafina y malla metálica interna, se compara con otro que sólo contiene parafina. Los dos prototipos se colocan al interior de agua a la temperatura de $90-91{ }^{\circ} \mathrm{C}$ (temperatura de ebullición del agua en Ayacucho), luego se registra la curva de enfriamiento de cada prototipo.

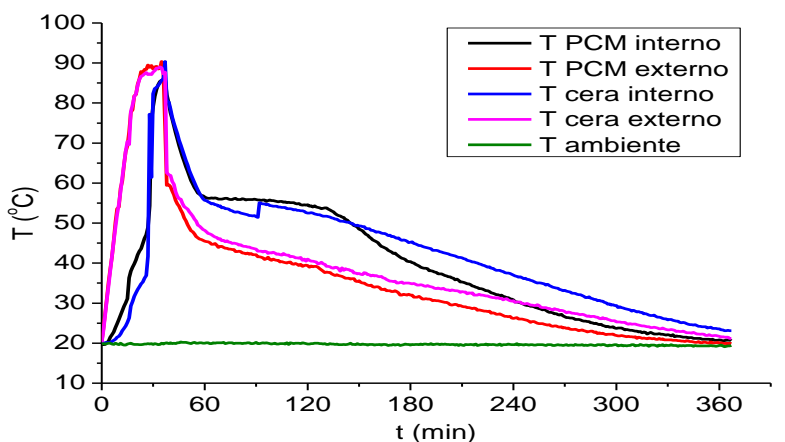

Fig. 9. Variación de la temperatura del prototipo de unidad del acumulador (T PCM interno y externo) y del prototipo que sólo contiene parafina. ( $T$ cera interno y externo)

La Fig. 9 muestra, que el prototipo de la unidad del acumulador se enfría más rápidamente que el prototipo que contiene sólo parafina sin malla metálica interna, ello debido a que la malla metálica aumenta la conductividad térmica del interior al exterior del cilindro lo que facilita mejor transferencia de calor al aire circundante; de manera que se ha mejorado la remoción de calor de la unidad cilíndrica del acumulador.

Cada unidad del acumulador térmico contiene 200 g de parafina y acumula energía térmica durante el periodo de incidencia de la radiación solar. Para estimar la energía acumulada en cada unidad, se asume la energía acumulada por calor sensible y latente en el intervalo de temperatura de $30^{\circ} \mathrm{C}$ a $60^{\circ} \mathrm{C}$. El resultado de la tabla $\mathrm{V}$ muestra que cada unidad acumula una energía de $40.91 \mathrm{~kJ}$, de manera que la energía disponible en un módulo del acumulador se estima en 490.94 kJ. Esta energía es la que entregará el acumulador al aire circundante, durante el proceso de enfriamiento, cuando cesa la incidencia de la radiación solar.

TABLA V

Energía Acumulada en cada unidad del módulo PCM.

\begin{tabular}{llll}
\hline Temperatura inicial & $\mathrm{T}_{\mathrm{i}}$ & 30 & ${ }^{\circ} \mathrm{C}$ \\
Temperatura de fusión de la cera & $\mathrm{T}_{\mathrm{fu}}$ & 53 & ${ }^{\circ} \mathrm{C}$ \\
\hline
\end{tabular}




\begin{tabular}{llll}
\hline Temperatura final & $\mathrm{T}_{\mathrm{f}}$ & 60 & ${ }^{\circ} \mathrm{C}$ \\
Masa de parafina & $\mathrm{m}$ & 0.2 & $\mathrm{~kg}$ \\
Calor latente de fusión de la cera & $\mathrm{L}_{f}$ & 123.506 & $\mathrm{~kJ} / \mathrm{kg}$ \\
Calor específico cera sólido & $\mathrm{C}_{\mathrm{ps}}$ & 2.66 & $\mathrm{~kJ} / \mathrm{kg}^{\circ} \mathrm{C}$ \\
Calor específico cera líquida & $\mathrm{C}_{\mathrm{pl}}$ & 2.34 & $\mathrm{~kJ} / \mathrm{kg}^{\circ} \mathrm{C}$ \\
Energía acumulada en una unidad & $\mathrm{Q}$ & 40.91 & $\mathrm{~kJ}$ \\
Energía acumulado en el módulo & $\mathrm{Q}_{\mathrm{T}}$ & 490.94 & $\mathrm{~kJ}$ \\
\hline (12 unidades) & & & \\
\hline
\end{tabular}

Nota: Los valores del calor latente de fusión y los calores específico [3]

Cuando se da la incidencia directa de la radiación solar sobre el colector del secador, inicia el proceso de calentamiento del colector, la temperatura de la placa metálica y la del acumulador instalado en ella van incrementándose, con el cual se va calentándose el aire del colector que luego ingresa a la cámara de secado, iniciando el proceso de secado del producto. En esta etapa el acumulador actúa como una carga que consume la energía y va acumulándola en el material PCM. Las figuras 10 y 11 muestran dicho proceso. Cuando se interrumpe o cesa la incidencia de la radiación solar, interviene el acumulador térmico cediendo energía al aire para mantener las condiciones apropiadas de secado durante un periodo adicional.

Del dimensionamiento energético realizado para el secado de papa, por ejemplo (ver TABLA IV), se encuentra que se requiere $385.2 \mathrm{~kJ}$ por hora para secar la papa de manera estacionaria y en las condiciones establecidas. Cuando cesa la radiación solar, la energía acumulada en el módulo que es de 490.94 kJ, permitiría disponer energía para calentar el aire por un intervalo de $1.3 \mathrm{~h}$ adicionales, considerando además la inercia térmica del propio secador este tiempo puede incrementarse.

\subsection{EVALUACIÓN DEL COMPORTAMIENTO TÉRMICO DEL SECADOR SOLAR CON Y SIN ACUMULADOR DE CAMBIO DE FASE}

Para determinar la influencia del acumulador solar térmico con material de cambio de fase (Parafina), en el secado del producto; se caracteriza la evolución temporal de la temperatura del aire a la salida del colector del secador cuando cesa la incidencia de la radiación solar sobre el colector. Este resultado es fundamental, dado que el aire a la salida del colector del secador es el aire caliente que ingresa a la cámara de secado, acelerando o retardando el proceso de secado del producto.

Las medias realizadas corresponden al secador cuando utiliza el acumulador solar térmico con PCM y cuando no utiliza el acumulador. En ambos casos se permite la incidencia de la radiación solar desde las 10 a.m. hasta las $2.30 \mathrm{pm}$, en la que se cubre el colector del secador con un material opacado, a partir del cual inicia el efecto del acumulador sobre el secado del producto.

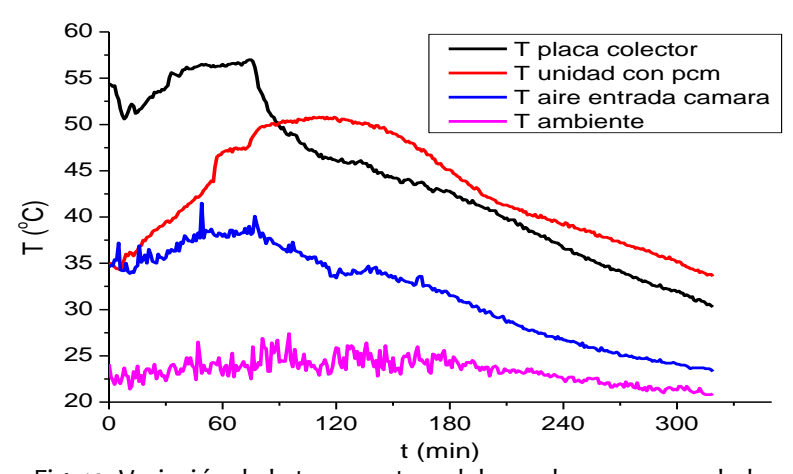

Fig. 10. Variación de la temperatura del secador con acumulador térmico con PCM, para una incidencia media de radiación solar desde las 10.00 am a 2:30 pm (que corresponde al minuto 75) $533 \mathrm{Wm}^{-2}$ (21/03/19).

Dada las condiciones ambientales en las que se realizó la prueba, la intensidad de radiación solar en ambos casos estuvo en el orden de 400 a $600 \mathrm{Wm}^{-2}$, de manera que no se alcanzó el potencial pleno del acumulador solar térmico con parafina.

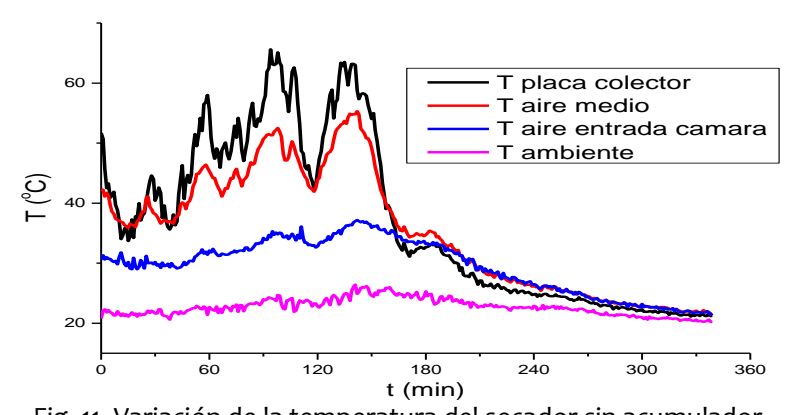

Fig. 11. Variación de la temperatura del secador sin acumulador térmico, para una incidencia media de radiación solar desde las 10.00 am a 2:30 pm(que corresponde al minuto 151) de $500 \mathrm{Wm}^{-2}(23 / 09 / 19)$

La Fig. 10 corresponde a la variación de la temperatura en el secador solar cuando se incorpora el sistema de acumulación térmica con PCM, en tanto que la Fig. 11, corresponde al secador sin PCM.

La Figuras 12 y 13, muestran la rapidez de variación de la temperatura del aire a la salida del colector. El aire que sale del colector es el aire caliente que ingresa a la cámara de secado, cuando no hay radiación solar, la temperatura del aire va disminuyendo de manera que la rapidez de enfriamiento es el indicador del grado de eficiencia del proceso de secado durante el tiempo adicional a la interrupción de la incidencia de la radiación solar. Para analizarla, se ha modelado dicha variación de manera lineal, de la Fig. 12 se obtiene una pendiente de $0.063{ }^{\circ} \mathrm{C} \min ^{-1}$ con un parámetro de correlación de 0.98 y de la Fig. 13, una pendiente de $0.081^{\circ} \mathrm{C} \min ^{-1}$ con un parámetro de correlación de 0.95 . 


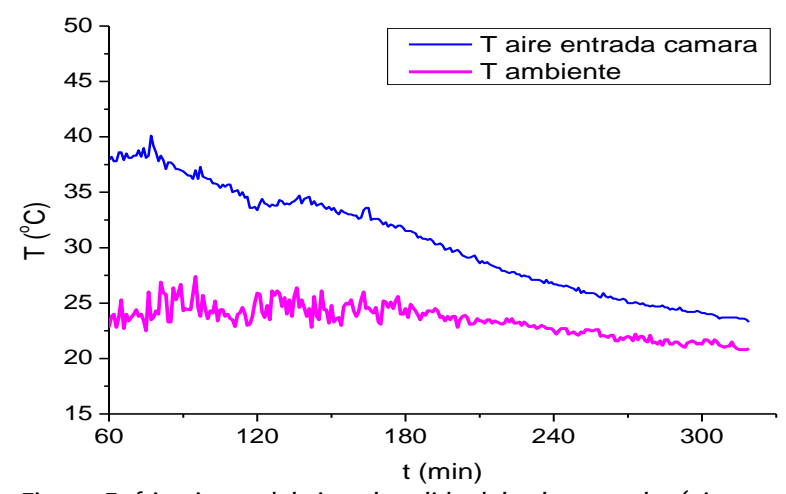

Fig. 12. Enfriamiento del aire a la salida del colector solar (aire que ingresa a la cámara de secado) a razón de $0.063{ }^{\circ} \mathrm{C} \mathrm{min}^{-1}$, cuando se utiliza el acumulador de cambio de fase (PCM) (21/03/19).

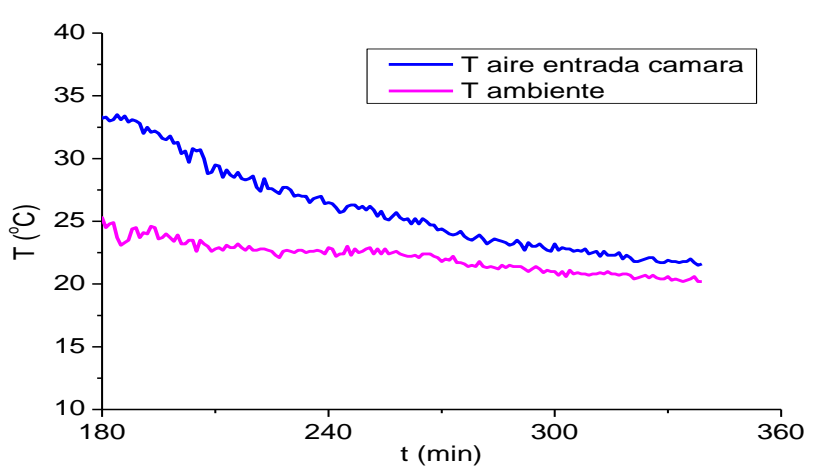

Fig. 13. Enfriamiento del aire a la salida del colector solar (aire que ingresa a la cámara de secado) a razón de $0.081{ }^{\circ} \mathrm{C} \mathrm{min}{ }^{-1}$, cuando no se utiliza el acumulador de cambio de fase (22/03/19).

\section{CONCLUSIONES}

- Las características térmicas de la parafina comercial, que permiten tomar en cuenta como un material de cambio de fase, medidos mediante el KD2 Pro, resultan: conductividad térmica de $\mathrm{k}=0.193 \pm 0.01 \mathrm{Wm}^{-1} \mathrm{~K}^{-1}$, difusividad térmica $\mathrm{D}=0.162 \pm 0.016 \mathrm{~mm}^{2} \mathrm{~s}^{-1}$ y calor específico volumétrico $\mathrm{C}=1.761 \pm 0.176 \mathrm{MJm}^{-3} \mathrm{~K}^{-1}$, en lo referente a la conductividad térmica concuerda por el reportado por Campos et al [3] que indica 0.20 Wm-1K-1 así como también Fernández [7], pero en lo referente al calor específico volumétrico Campos obtiene un calor específico de $2660 \mathrm{~J}(\mathrm{Kg})-1 \mathrm{~K}-1$ en fase sólida; mientras que el que calculamos tomando en cuenta la relación $\mathrm{C}=\rho \mathrm{c}_{\mathrm{p}}$ con $\rho$ la densidad medida de la parafina, resultó ser igual a $2111 \mathrm{~J}(\mathrm{Kg})-1 \mathrm{~K}-1$ que difiere en un $20 \%$, sin embargo el calor específico que reporta Vidal [5] es de $2100 \mathrm{~J}(\mathrm{Kg})-1 \mathrm{~K}-1$. Estos resultados nos muestran que la cera (parafina) comercial distribuida en Ayacucho si tiene las características térmicas apropiadas para ser considerado un material de cambio de fase.

- La gráfica de la Fig. 9 muestra que el comportamiento de las unidades de acumulación cilíndrica con parafina y mallas o aletas internas, intercambia calor con mayor rapidez con el exterior que las unidades de acumulación que tienen sólo parafina. Las figuras mencionadas muestran una diferencia de temperatura en el orden de 5 oC luego de un periodo de 5 horas.

- El comportamiento térmico del sistema de acumulación solar con PCM (parafina) mostrada en la Fig. 10, establece que la temperatura del aire a la entrada a la cámara de secado se ha mantenido en el orden de $30{ }^{\circ} \mathrm{C}$, por un periodo adicional de $2 \mathrm{~h}$ sin la intervención de la radiación solar. En tanto que en el secador, que no utiliza el acumulador de cambio de fase, la temperatura decae más rápidamente (Fig. 11). La Fig. 12 y 13 caracterizan la rapidez de variación de la temperatura del aire que entra a la cámara de secado, mostrando un decaimiento de $0.06 \mathrm{oC}$ min-1 para el que usa PCM y de 0.08 oC min-1 cuando no usa PCM.

Las conclusiones del presente trabajo son:

- Se dimensiona y optimiza un prototipo de un acumulador solar térmico con parafina comercial como material de cambio de fase, se caracteriza por ser modular compuesto de 12 cilindros metálicos de aluminio reciclado que contiene 2.4 kg de parafina y que para mejora la conductividad térmica de la parafina $\left(0.198 \mathrm{Wm}^{-1} \mathrm{~K}^{-1}\right)$ se incorpora al interior del cilindro una malla metálica de aluminio con aletas que optimiza la transferencia de calor homogénea de la parafina al aire. Cada unidad del acumulador contiene una energía de $40.91 \mathrm{~kJ}$ por calor sensible y latente cuando se calienta desde $30{ }^{\circ} \mathrm{C}$ a $60{ }^{\circ} \mathrm{C}$; la temperatura de fusión de la parafina está entre $53{ }^{\circ} \mathrm{C}$ y $56^{\circ} \mathrm{C}$, con un calor específico volumétrico de $1.762 \mathrm{MJm}^{-3} \mathrm{~K}^{-1}$ (ver TABLA III).

- La concepción del acumulador térmico, se orienta a atender la demanda energética adicional para optimizar el proceso de secado en un secador solar familiar de mediana temperatura, de tipo cabina e indirecto. Mediante el uso del acumulador térmico solar, se logra aumentar el tiempo de secado en horas adicionales al cese de la intervención de la radiación solar por un periodo de hasta $2 \mathrm{~h}$, manteniendo la temperatura del aire de la cámara de secado superior a $30{ }^{\circ} \mathrm{C}$, lo que optimiza el proceso de secado.

- Para aumentar mayor tiempo de secado sin intervención solar, es necesario agregar mayor cantidad de unidades del acumulador, lo que permite el carácter modular del diseño propuesto.

\section{AGRADECIMIENTOS}

Agradecemos a la Universidad Nacional de San Cristóbal de Huamanga, por hacer posible el presente trabajo de investigación. 


\section{REFERENCIAS}

[1] M. Escobar, S. Cuervo y S. Rincon. "Metodología para el diseño de un módulo de acumulación térmica usando materiales de cambio de fase (PCM) para un secador solar de túnel", Revista UIS Ingenierías, vol. 17, no. 1, pp. 9-20, nov., 2017.

[2] A. I. Glogower. "Sistema de almacenamiento de energía térmica en el cambio de fase de materiales para un secador solar tipo Hohenheim", tesis de grado, Universidad de los Andes Colombia, 2015.

[3] A. Campos, J. Gonzalo, T.V. Terés, A. Bandos, M. García, L.M. López y J. M. Salac. "Design of a finned plate latent heat thermal energy storage system for domestic applications", Energy Procedia, vol. 48, pp. $300-308,2014$.

[4] Agroalimentando, Ciencia y tecnología aplicadas a la agricultura y a la alimentación. "Acumulador de energía térmica para secado de agroproductos".

Available:
[5] B. Vidal. "Modelización del cambio de fase sólido líquido. Aplicación a sistemas de acumulación de energía térmica", tesis doctoral, Universitat Politiecnica de Catalunya, 2007.

[6] L. Blanco, L. Valldecabres. Guía para el desarrollo de proyectos de secado solar en comunidades rurales. Madrid: Energía Sin Fronteras 2016.

[7] W. Fernández. "Análisis del comportamiento del perfil de temperaturas de material de cambio de fase (PCM) en el almacenamiento de energía solar utilizando herramientas computacionales", tesis de pregrado, Universidad Autónoma del Caribe, Colombia, 2012.

\section{(c) (1)}

Los artículos publicados por TECNIA pueden ser compartidos a través de la licencia Creative Commons: CC BY 4.o. Permisos lejos de este alcance pueden ser consultados a través del correo revistas@uni.edu.pe 